\title{
A Review of Static Behavior Research
}

\author{
Bin Wang, Luyao Chen, Zhifei Wang*, Zhenlu Zhang \\ College of Physical Education and Health Science, Zhejiang Normal University, \\ Yingbin dadao, Jinhua City, Zhejiang Province, China \\ *Correspondence Author
}

\begin{abstract}
By using the methods of literature and logical analysis, this paper expounds the related research of static behavior and analyzes the development trend of static behavior research. The related research on static behavior includes the research on the epidemic situation, influencing factors, measurement methods, epidemiological relationship and intervention means of static behavior. The key is to study the influence of static behavior on human health, many studies show that static behavior is closely related to cardiovascular diseases, cancer, obesity, bone density, vision, neuroendocrine, depression and happiness. Most of them use questionnaires to measure static behaviors, while only a few use objective instruments to measure static behaviors. At the same time, it puts forward several development trends of static behavior research: comprehensive investigation and research, specialization of measurement tools, deepening of health impact research and diversification of intervention means. It provides a reference for further study of static behavior.
\end{abstract}

Keywords: Intestinal flora, Obesity, Fat, Energy.

\section{Introduction}

At present, the amount of necessary activities in people's daily work and life has decreased, and the way and content of physical activities have also changed, Static life has become a common way of life. However, people have fully realized the influence of static behavior on health, and all countries strongly advocate people of all ages to strengthen moderate and high-intensity physical exercise to promote people's health. Static behavior has also become a research hotspot, at present, the research status and development trend of static behavior are mostly aimed at a certain group or a related point, and there is little research on the overall research status and development trend of static behavior. Therefore, the overall research status and development trend of static behavior are described. The research system of static behavior is relatively complete, but there are still aspects that can be further studied in its related research, for example, most of the current research on static behavior is aimed at local areas or specific people, and there is little research on the whole, and the "localization" of the questionnaire in the measurement of static behavior needs constant research; And the most important intervention means of static behavior also needs more in-depth study. On this premise, this paper discusses the current research status and development trend of static behavior, aiming to systematically sort out the previous research and provide a reference train of thought for the related research in the future.

\section{Research Status of Static Behavior}

\subsection{The Concept of Static Behavior}

The research on the concept of static behavior is simply equivalent to lack of physical activity from the early 1990s, In 2010, Owen et al[1], defined static behavior as some negative physical behaviors with little body movement and energy consumption close to resting metabolic rate, such as sitting or lying watching TV, working, studying or resting. By 2012, the concept was redefined by the Static Behavior Research Network (SBRN) as: all behaviors whose energy consumption does not exceed 1.5 metabolic equivalent when awake, sitting or lying [2]. Then, in 2017, the static behavior is defined as any behavior in which energy consumption is less than or equal to 1.5 metabolic hours (METs) in awake state, including sitting, reclining or lying; Sitting is defined as the posture in which an individual holds his back upright and supports his weight with his hips instead of his feet, lying is in a horizontal position on the supporting surface, and lying on his back lies between sitting and lying [3], [4]. In a word, static behavior is not equal to lack of physical activity, nor is it the simple opposite of physical exercise, it is a special behavior independent of low physical activity, with more emphasis on low energy consumption behavior.

\subsection{Research Status of Influencing Factors of Static Behavior}

\subsubsection{Physiological factors}

A large number of studies show that the physiological factors of human body have great influence on the time of static behavior. If some studies have found that with the increase of age, children's static behavior time in front of the screen increases. A longitudinal survey by Hardy et al, shows that with the increase of teenagers' age, the aggravation of their schoolwork tasks and the change of their entertainment style lead to the increase of their static behavior time [5]. According to the research of Clark et al [6], women spend more time watching TV than men, which leads to women's static behavior time being longer than men. Therefore, the individual's age, gender, and health status will affect the occurrence of static behavior.

At the same time, the individual's physical health will also have a certain impact on static behavior. For example, research by Van Alphen et al [7], shows that elderly people with dementia have more static behaviors and less physical activity due to diseases. The investigation results of Hendrickxe et al[8], also show that the walking speed of stroke patients is slow, which affects physical activity and thus has more static behaviors. Drenkamp et al [9], showed that patients with two chronic diseases showed more static behaviors. On the whole, the individual's own physiological condition has great influence on static behavior. 


\subsubsection{Social background factors}

People are a group living in a society, Social background and surrounding environment will also have certain influence on static behavior. The study of economic status shows that the scale of social economic status is inversely proportional to static behavior. Similarly, static behavior shows a certain correlation with people's occupations, Occupations require people with more activities to carry out activities in order to complete their professional tasks, which objectively reduces the occurrence of static behavior. In addition, different educational levels have different influences on static behaviors, The research results of Lin et al[10], show that the elderly with low educational level, living alone and not working full-time have more static behaviors, especially watching TV, so they are less likely to engage in moderate to severe physical activities and muscle strengthening exercises.

\subsubsection{Environmental factors}

The surrounding living environment has a great influence on people, and so does the static behavior. First of all, the influence of intimate relationship shows great correlation, Parents, teachers and friends' encouragement, encouragement and leading by example in intimate relationship can improve teenagers' enthusiasm for participating in sports activities to a certain extent. Among them, good friends have the most profound influence. Besides intimacy, sports venues, neighborhood environment and natural environment near the residence have important influences on people's static behavior. Teenagers spend most of their time in school, many objective measurement studies have found that school environment has a significant impact on the overall static behavior, The higher the score index of school sports environment, the lower the overall static behavior time of students. For adults, the working environment also affects their static behavior to a certain extent.

\subsection{Research Status of Static Behavior Measurement}

At present, the measurement methods of static behavior mainly include questionnaire measurement, diary survey and objective measurement. Among them, the questionnaire of static behavior is divided into self-reporting and proxy-reporting, the commonly used self-reporting questionnaires mainly include Sedentary Behavior Questionnaire (SBQ) [11], Marshall Sitting Behavior Questionnaire (MSBQ) [12], The Occupational Sitting and Physical Activity Questionnaire (OSPAQ) [13], Adolescent Sedentary Activity Questionnaire (ASAQ). Objective measurement methods mainly include acceleration sensor measurement method, double standard water method and indirect heat measurement method.

SBQ is used to measure the time of nine static behaviors in daily life, including watching TV, playing video games, sitting and listening to music, sitting and answering the phone, doing homework or office work, sitting and reading, playing musical instruments, making art and crafts, and traffic sedentary behavior. Compared with SBQ, MSBQ focuses on comprehensive measurement of sit-in time, including traffic time, work sit-in time, watching TV time, using computer time and leisure static time (excluding watching TV time). However, the measurement effect of MSBQ in weekend is not as good as that in week, which is also a problem to be solved in future research. OSPAQ is a questionnaire that measures physical activity and static behavior at the same time, the questionnaire investigates the activities such as sitting, standing, walking and heavy physical labor from Monday to Friday. Among the current measuring tools, ASAQ is one of the few measuring tools suitable for teenagers, and the measurement indicators used are suitable for teenagers' behavior patterns.

\subsection{Investigation and Study of Static Behavior}

2.4.1 Investigation on the static behavior of children and adolescents

The investigation and research on the static behavior of children and adolescents at home and abroad show that the static behavior time is too long. Especially the static behavior time in front of the screen is too long. For example, Guthold et al[14],found that more than one third of students spend more than three hours in their leisure activities every day according to the data of 34 countries around the world. According to the research report of China Health and Nutrition Survey (CHNS) [15], from 1997 to 2006, children aged 6-18 in China showed an obvious increasing trend in time before screen and time for homework among people of all ages in different regions. Chinese scholars have begun to realize the adverse effects of static behavior on children's physical and mental health [16].

He Jian et al, research on the static behavior of middle school students in Henan Province shows that it is very common for middle school students in Henan Province to arrange their spare time by static activities [17]. Research by Zhu Shanshan et al [18], shows that the average daily sedentary time of students before and after puberty is $6.35 \mathrm{~h}$ hours.

\subsubsection{Status quo of adult static behavior investigation}

Research by Chen Xiaorong and others in 2010 showed that the regular exercise rate of adults in China was $11.9 \%$ (95\% CI: $10.5 \%$ 13.3\%) [19]. Static behavior has become the main life style of different people in the awake period, especially in leisure time. Bauman et al [20], investigated adults aged $18-65$ in 20 countries around the world, and found that the average static behavior time of the investigated population was $5 \mathrm{~h} / \mathrm{d}$, and there was a big gap between countries. In China, adults spend about 3.5 hours on static behavior in their leisure time, of which watching TV takes up 2/3 of the time [21]. A study of Shanghai residents by Gu Kai et al [22], shows that more than half of Shanghai residents spend more than $5 \mathrm{~h} / \mathrm{d}$ in static behavior every day.

According to the survey statistics of more than 50,000 people in many countries around the world, about $60 \%$ of the elderly reported that their static behavior time was $>4 \mathrm{~h} / \mathrm{d}$, and this value increased sharply to $8.5 \mathrm{~h} / \mathrm{d}$ when measured by objective method [23]. In America, teenagers aged 16-19 and older people aged $>60$ have the longest static behavior time [24]. 


\subsection{The Research Status of the Influence of Static Behavior on Human Health}

2.5.1 The influence of static behavior on human physiological health

Long time of static behavior has a great impact on human physiological health, in 2010, the American Cancer Society conducted a 14-year follow-up study on 123,216 adults, and found that the death risk of women who sat for 6 hours a day was $34 \%$ higher than that of women who sat for 3 hours, while the death risk of men who sat for a long time increased by $17 \%$. In the 12-year follow-up study of more than 90,000 women aged 50.79, scholars found that compared with the shortest group (sitting for more than 11 hours a day), the risk of cardiovascular disease, heart disease and tumor increased by $13 \%, 27 \%$ and $21 \%$ respectively. In the following studies, it has been shown that too long static behavior will increase the prevalence of cardiovascular diseases, cancer, metabolic syndrome, obesity, diabetes, vision and so on. At the same time, too long static behavior has an adverse effect on the growth of adolescent bone, resulting in the reduction of bone content and the peak bone density; Too long static behavior will also lead to the decline of physical fitness.

\subsubsection{The influence of static behavior on human mental health}

At the same time, a large number of studies have shown that static walking not only has an impact on human physical health, but also has a certain impact on human mental health. The static behavior in front of the screen is too long, the mental health is worse, and the risk of depression is increased. However, watching TV for a long time is negatively correlated with positive emotion, Long-term Internet access, long-term video games, excessive use of Internet and excessive use of games are negatively correlated with life satisfaction, personal happiness index and positive emotion. Bartel and others found that the longer teenagers use computers, the shorter their sleep time. Excessive use of technology products by teenagers will increase sleep problems, reduce sleep time and delay bedtime. Teenagers who play in front of the screen for more than one hour have a significant correlation with attention, learning consciousness, academic achievement and school relationship. Similarly, studies have shown that regardless of the overall physical activity, the elderly spend a lot of time on static behavior, which will lead to an increase in the probability of cognitive impairment.

\subsection{Research Status of Static Behavior Intervention}

The specific measures of static behavior intervention are often complex and diverse, according to Wang Lei [25], Jia Yingnan, etc., the specific intervention measures included in the literature are classified into three levels: organization management, material environment and individual.

\subsubsection{Organization and management}

From the specific content, the measures taken at the organizational management level include policy support and the formulation of new implementation plans. Jason et al, found that most middle school students have not realized the harm of sedentary behavior to their own health. Therefore, it is very important to intervene the static behavior of young students. Schools are also an important place to promote students' physical and mental health, so schools should also be the main place for young students to intervene in static behaviors. At present, all kinds of schools at all levels in china carry out sunshine sports and exercise for one hour every day, all of which are aimed at giving students more time to participate in sports every day and reducing the time of students' static behavior. Higher-level education departments are constantly emphasizing the importance of sports activities for teenagers' physical and mental health, demanding to carry out sunshine sports activities, opening up physical education class and so on. All of them play a certain intervention role in static behavior.

\subsubsection{Physical environment}

Intervene static behavior through material environment, which includes the material environment of campus and family for teenagers. School performance is to improve sports venues and facilities, fully staffed with physical education teachers, at the same time, open a good physical education class and implement sunshine sports activities. At home, the arrangement and placement of fitness facilities in the home and community play an important role in the intervention of static behavior.

For professionals, one of the most widely used measures is to install and use a sitting-standing desk, which has different structural styles, but its basic function is that the height of the desktop can be adjusted, so that users can conveniently switch between sitting and standing positions to work; Another measure worthy of attention is to install software that regularly reminds you to interrupt your sitting posture and stand up and move on your office computer; Other environmental strategies include various measures aimed at interrupting employees' sedentary and increasing walking, such as setting up central public printers and garbage bins in public offices.

\subsubsection{Personal}

At the personal level, there are mainly face-to-face guidance on knowledge and skills related to sedentary risk intervention, guiding and encouraging telephone calls and emails, goal setting and information feedback, etc. In one study, individual intervention suggestions were customized by expert software based on personal survey information. It is worth noting that focusing on the theme of reducing sitting posture, and discussing and implementing specific interventions by participants are generally valued, this method is obviously more suitable for the specific conditions of different workplaces, and is convenient for participants to implement, thus being easy to operate and effective.

KIKUCHI et al[26], also found that improving the social participation of the elderly can increase their physical activity and reduce their static behavior. Boulton et al[27], regularly send incentive information to the elderly by using smart phones and smart watches, which makes the elderly form a long-term belief in physical activity and changes their habit of having more static behaviors. 


\section{The Development Trend of Static Behavior Research}

\subsection{Comprehensive Investigation and Study}

At present, most of the researches on the status quo of static behavior investigation are local studies in different regions or different groups of people, and there are few researches on the whole, but there must be some differences between the local investigation and the whole investigation. Therefore, it may be more extensive in the following investigation and study. At the same time, a large part of static behavior investigation is aimed at the present situation of teenagers' static behavior in front of the screen, which does have great hidden dangers, but there are also great hidden dangers in the static behavior in front of the screen among middle-aged and old people, In view of this phenomenon, we should pay more attention to the static behavior of middle-aged and old people and do some investigation and research before we can effectively intervene and promote the health of middle-aged and old people.

\subsection{Specialization of Measuring Tools}

At present, the measurement of static behavior includes questionnaire survey, double standard water method, indirect calorimetry, diary survey and so on, among which questionnaire survey is divided into self-report questionnaire and proxy report questionnaire. The objective measurement of static behavior is basically the same as the measurement of physical activity, including acceleration sensor measurement, double standard water measurement and indirect heat measurement. At present, the static behavior measurement lacks the "gold standard", and there is still no recognized optimal method for static behavior measurement.

First of all, most of the current survey questionnaires come from abroad, so language compatibility and stability of data collection are still a big challenge in cross-cultural transformation. Therefore, the development of static behavior measurement questionnaire should consider cultural differences and select the most common measurement indicators in the crowd to build an effective tool.

Secondly, the objective measurement method of static behavior is the same as the measurement method of physical activity, but we can know that static behavior is different from physical activity from the constant updating of the concept of static behavior, so we can continue to study the specialization of objective measurement method of static behavior in later studies. Make the measurement data of later research more accurate, and make the research more targeted.

\subsection{In-depth Study of Health Impact}

The influence of static behavior on human health is divided into two aspects: physiological and psychological, At present, there are many studies on the influence of static behavior on human health in medicine, among which there are many studies on cardiovascular and obesity, and relatively few studies on the influence of other diseases, The research on other diseases in the future can be continuously deepened to understand the influence of static behavior on human health more comprehensively.

The research on the influence of mental health involves depression, sleep, happiness, academic performance, cognitive ability and so on. The most prominent one is the research on the influence of depression and sleep. According to the definition of static behavior, the time of static behavior of primary and secondary school students is definitely too long, and only a small amount of physical education class every day and the rest of class exercises belong to static behavior except sleeping, in view of this phenomenon, more research should be done on static behavior corresponding to learning, because for teenagers, the effect of learning is very important. At the same time, the impact on cognitive ability is currently discussing the correlation between Cognitive aging of the elderly, and cognitive ability is equally important for teenagers.

Finally, many studies have discussed a series of diseases caused by the decline of physical activity caused by excessive static behavior. However, static behavior is not a simple lack of physical activity, and later research should be more focused on the impact of static behavior itself on human health.

\subsection{The Diversity of Interventions}

Relatively speaking, teenagers spend most of their time at school and spend the rest of their time in classrooms except for the collective extracurricular activities arranged by the school, which belongs to the category of static behavior. It is impossible to completely change the problem that static behavior takes too long only by relying on physical education class and sports activities between classes. At the same time, some studies show that too long static behavior will not only affect the physical health of the body, but also affect the academic performance and learning consciousness of teenagers. Therefore, in view of this phenomenon, whether there is a more effective way to solve this problem from the root. It also requires us to diversify the research on intervention means in future research.

Similarly, the existing intervention means of static behavior, besides the arrangement of sports activities in school, the other means of increasing peripheral facilities, ideological education, etc, are guiding means, lacking some mandatory means to intervene. Or whether there are some methods that can be done at home without excessive exercise consumption, just as most of the static behavior time at home is due to watching TV and playing mobile phones, then whether we can have some interventions that do not conflict with watching $\mathrm{TV}$ and playing mobile phones can not only improve the static behavior time, but also do not need to put down what we are doing.

Most of the existing interventions are aimed at teenagers, thus ignoring the intervention on the static behaviors of young and middle-aged people, However, their physical functions have declined and their burdens have increased, which makes it more necessary to intervene in the static behaviors, which is conducive to promoting their health and family happiness. Therefore, in the future, a research trend may tend to interfere with the static behavior of young and middle-aged people. 
Finally, combined with the existing research, we can intervene the static behavior under the guidance of sports activities and the optimization and integration of medical and health services, and make a certain contribution to the health of the organism, thus improving the overall health level of human beings.

\section{Concluding Remarks}

There are many researches on static behavior, including the definition of concept, influencing factors, measurement of static behavior, investigation of epidemic situation, influence on human health and intervention of static behavior. The results of static behavior research are constantly transformed and applied to practice. After systematically combing the research status of static behavior, it also analyzes and discusses the possible development trend of static behavior research from several aspects-comprehensive investigation and research, specialization of measurement tools, deepening of health impact research, diversification of intervention means and so on. I also believe that the harm of static behavior to human health can be reduced more effectively through static behavior intervention after the continuous improvement of research.

\section{References}

[1] Owen N, Healy GN, Matthew s CE, et a1. Too much sitting: the population health science of sedentary behavioral [J]. Exerc Sport Sci Rev, 2010, 38(3): 105-113.

[2] Tremblay M. Reply to the discussion of "letter to the editor: standardized use of the terms sedentary and sedentary behaviours"- sitting and reclining are different states [J]. Appl Physiol Nutr Metab, 2012, 37(6):1257.

[3] Tremblay MS, Aubert S, Barnes JD, et al. Sedentary behavior research network (SBRN)-terminology consensus project process and outcome [J]. Int J Behav Nutr Phys Act, 2017, 14(1): 75.

[4] Qiu Dayong, Li Huinan, Zhang Yan. Research on the Influence of Static Behavior on Adolescent Students' Physical Health [J]. Sports teachers and friends, 2017, 40(04): 57-59.

[5] Wang Weiping. Study on the related factors affecting teenagers' static behavior [J]. Exam weekly, 2018, (80): 36.

[6] Bronwyn Kay Clark, Takemi Sugiyama, Genevieve N. Healy, Jo Salmon, David W. Dunstan, Jonathan E. Shaw, Paul Z. Zimmet, Neville Owen. Socio -Demographic Correlates of Prolonged Television Viewing Time in Australian Men and Women: The AusDiab Study [J]. Journal of Physical Activity and Health, 2010, 7(5): 595601.

[7] VAN ALPHEN H J, HORTOB GYI T, VAN HEUVELEN M J. Barriers, motivators, and facilitators of physical activity in dementia patients: a systematic review [J]. Arch Gerontol Geriatr, 2016, 66: 109-118.

[8] HENDRICKX W, RIVEROS C, ASKIM T, et al. Identifying factors associated with sedentary time after stroke. Secondary analysis of pooled data from nine primary studies [J]. Top Stroke Rehabil, 2019, 26(5): 327-334.

[9] DRENKAMP S, MESTERS I, VOS R. Synergistic effects of six chronic disease pairs on decreased physical activity: the SMILE cohort study [J]. Biomed Res Int, 2016, 2016: 9427231.

[10] LIN C Y PARK J H, HSUEH M C. Prevalence of total physical activity, muscle-strengthening activities and excessive TV viewing among older adults; and their association with sociodemographic factors [J]. Int J Environ Res Public Health, 2018, 15(11): 1-9.

[11] ROSENBERG D E, NORMAN G J, WAGNER N, et al. Reliability and validity of the sedentary behavior questionnaire (SBQ) for adults [J]. Journal of Physical Activity \& Health, 2010, 7(6): 697-705.

[12] MARSHALL A L, MILLER YDBURTON N W, BROWN W J. Measuring total and domain-specific sitting: a study of reliability and validity [J]. Medicine \& Science in Sports \& Exercise, 2010, 42(6): 1094-1102.

[13] CHAU J Y, VAN DER PLOEG H P, DUNN S, et al. Validity of the occupational sitting and physical activity questionnaire $[\mathrm{J}]$. Medicine \& Science in Sports \& Exercise, 2012, 44(1): 118-125.

[14] Guthold R, Cowan M J, Autenrieth C S, et a1. Physical activity and sedentary behavior among Schoolchildren: a 34-country comparison[J]. The Journal of Pediatrics, 2010, 157(1): 43-49.

[15] Cui Z, Hardy L L, Dibley M J, et a1. Temporal trends and recent correlates in sedentary bchaviours in Chinese children [J]. Int J Behav Nutr Phys Act, 2011, 8(1): 93.

[16] Li Haiyan, Chen Peijie, Zhuang Jie. Correlation between physical activity and physical health of adolescents in Shanghai $[\mathrm{J}]$. Shanghai Preventive Medicine, 2011, 23(4): 145-149.

[17] He Jian, Yang Biansheng, Zhong Ya, Peng Yongping, Liang Zhenshan. Research and Analysis on Static Activities Related Behaviors of Urban Middle School Students in Henan Province [J]. Chinese Journal of School Health, 2008, (05): 412-414.

[18] Zhu Shanshan, Liu Yunfa, Cai Xiaohong, et al. A survey of daily behavior patterns and physical condition of students in different stages of puberty [J]. Journal of Guangzhou Sport University, 2014, 34(6): 83-87.

[19] Chen Xiaorong, Jiang Yong, Wang Limin, Li Yichong, Zhang Mei, Hu Nan, Zhao Wenhua. Analysis of Chinese adults' amateur exercise and amateur static behavior in 2010 [J]. Chinese Journal of Preventive Medicine, 2012, (05): 399-403.

[20] Bauman A, Ainsworth BE, Sallis JF, et al. The descriptive epidemiology of sitting: a 20-country comparison using the international physical activity questionnaire (IPAQ) [J]. Am J Prev Med, 2011, 41(2): 228-235.

[21] Ye Sunyue, Claire Kuo. Prevalence, Trend and Influencing Factors of Physical Exercise and Static Behavior of Chinese Adults [J]. Journal of Capital Institute of Physical Education, 2016, 28(4): 365-369.

[22] Gu Kai, Shen Medal, Sun Jianping, etc. An analysis of meditation style and physical exercise of community residents in Shanghai and its influencing factors[J]. Shanghai Journal of Preventive Medicine, 2002, 14(9): 444-446. 
[23] Harvey JA, Chastin S, Skelton DA. Prevalence of sedentary behavior in older adults: a systematic review [J]. Int J Environ Res Public Health, 2013, 10: 6645-6661.

[24] Matthews CE, Chen KY, Freedson PS, et al. Amount of time spent in sedentary behaviors in the United States, 2003-2004 [J]. Am J Epidemiol, 2008, 167: 875-881.

[25] Wang Lei, Duan Zicai. Reducing sedentary at work: research progress on intervention methods and effects [J]. Chinese Journal of Sports Medicine, 2020, 39(02): 145-151.

[26] KIKUCHI H, INOUE S, FUKUSHIMA N et al. Social participation among older adults not engaged in full-or part-time work is associated with more phy sical activity and less sedentary time[J]. Geriatr Gerontol, 2017, 17(11): 1921-1927.

[27] BOULTON E. HAWLEY-HAGUE H FRENCH D P, et al. Implementing behaviour change theory and tech niques to increase physical activity and prevent functional decline among adults aged 61-70: the prevent IT project [J]. Prog Cardiovasc Dis, 2019, 62(2): 147-156.

\section{Author Profile}

Bin Wang, professor, master tutor, School of Physical Education and Health Sciences, Zhejiang Normal University. Research direction: humanities and sociology of sports.

Luyao Chen, postgraduate student of Zhejiang Normal University. Research direction: sports training.

Zhifei Wang, postgraduate student of Zhejiang Normal University. Research direction: physical education.

Zhenlu Zhang, postgraduate student of Zhejiang Normal University. Research direction: physical education. 